\title{
Research and Implementation of Complex Failure Real-time Calculation Module in EMTP-based Relay Protection Tester
}

\author{
Jianshi Bai ${ }^{1, a,{ }^{\star}}$, Fang Shen ${ }^{1, b}$, Yi Liang ${ }^{1, \mathrm{c}}$, Jing Gao ${ }^{1, \mathrm{~d}}$, Zhentao Han ${ }^{1, \mathrm{e}}$, Yang \\ $\mathrm{Bai}^{2, \mathrm{f}}$ \\ ${ }^{1}$ Economic Research Institute of State Grid Liaoning Electric Power Co., Ltd., Shenyang, Liaoning, \\ China \\ ${ }^{2}$ Jinzhou Power Supply Company of State Grid Liaoning Electric Power Co., Ltd., Jinzhou, \\ Liaoning, China \\ alunaticbjs89@126.com
}

Keywords: Relay Protection Tester, EMTP, Complex Failure Real-time Calculation Module

\begin{abstract}
At present, the report format of relay protection tester's traditional simulating calculation is not uniform, and can only provide a simple failure analysis of the power grid. As for the RTSD that can carry out complex analysis, theoretical requirements are too high and the price is very expensive. Aiming at this problem, this paper presents an EMTP-based complex failure real-time calculation module for relay protection tester. By building power system relay protection commonly-used transmission line model in EMTP, rewritten program embedded under DOS environment, writing the program to automatically generate COMTRADE standard format files and other researches, to achieve a low-cost computing module that can be used in relay protection test for complex failure calculation, can operate easily and can generate a unified reporting format.
\end{abstract}

\section{Introduction}

The failure of the power system is becoming more and more complicated, and the traditional relay protection tester is based on the principle of hand calculation. The principle of hand calculation can only calculate simple fault of the system. Therefore, the traditional relay protection tester has been unable to calculate the increasingly complex power system failure and multi-point failure.

The new calculation module proposed in this paper is based on the simulation system EMTP. Through the research and development of the new calculation module, it is better than the traditional use of relay protection tester and RTDS in practical application. The new computing module can not only fully allow the real-time information transmission with the actual protection device, but also can achieve complex fault model calculation and analysis that the traditional simulation calculation cannot complete. One important factor is that the price of the new computing module is significantly lower than the well-performed but expensive RTDS. Under the premise that the performance should meet the requirements of the relay protection test, the product cost is improved. In this paper, the new calculation module and the traditional relay protection tester simulation and RTDS were compared to illustrate its advantages.

\section{Traditional simulation calculation principle}

The traditional relay protection tester simulation calculation program is running under Windows, and capable of a certain amount of real-time information exchange. Its principle is based on manual calculation, only a simple short circuit calculation can be performed such as single-phase grounding, two-phase grounding, two-phase short circuit and three-phase short circuit and so on. The premise of the manual calculation formula is that the system failure is a simple fault. The formula can be applied directly if it's a symmetry failure. But if it's an asymmetric fault, then the symmetrical component should be transformed into the symmetrical component of zero sequence, positive sequence and negative sequence, and then the corresponding mathematical formula is selected 
according to the system fault type. The short circuit current is calculated by the formula, and finally the waveform is drawn according to the instantaneous value of the current. For example: the system occurs two-phase short-circuit fault (BC).

The principle of the test is manual calculation. Using the data to calculate the short circuit impedance, and finally draw the fault current-voltage waveform. The drawing of the waveform is based on equations (2-1) and (2-2):

$$
\begin{aligned}
& i=\sqrt{2} I \sin (\omega t+a) \\
& u=\sqrt{2} U \sin (\omega t+a)
\end{aligned}
$$

Where $\mathrm{a}$ is the short-circuit impedance phase angle, $\mathrm{I}$ and $\mathrm{u}$ stand for the current and instantaneous voltage value, I and U stand for the RMS of current and voltage.

Short circuit current, system voltage and other conditions can be used in two-phase short-circuit manual calculation method to obtain short-circuit impedance. The method is that by setting the boundary condition and converting to the symmetrical component, and finally get the formula (2-3), (2-4):

$$
\begin{aligned}
& \dot{I}_{b}=j \frac{\dot{U}}{Z_{(1)}+Z_{(2)}} \\
& \dot{I}_{c}=-j \frac{\dot{U}}{Z_{(1)}+Z_{(2)}}
\end{aligned}
$$

a in equations (2-1) and (2-2) can be obtained from the short-circuit impedance.

After the test is ready, click to start the test, the computer carries out the calculation, and ultimately through the traditional manual simulation calculation principle, drawing fault waveform. Therefore, we can see the traditional simulation is performed by simple model and formula, the formula cannot be applied to complex power system fault calculation. For example: two short circuit at the same time, a short circuit and a break at the same time or transformer gate failure cannot use this traditional relay protection tester simulation calculation.

\section{New calculation module performance and testing process}

\subsection{New calculation module performance analysis}

The new calculation module is based on independent-developed EMTP simulation software. The main reason for choosing EMTP is that it has a number of common models for electric systems. Users can build their own system network models through the software, and control and analyze their models. The software can not only simulate and analyze the simple fault and symmetrical fault of the system, but also can simulate the multi-point composite fault and asymmetry fault.

The new calculation module utilizes the EMTP component model library to build a rich common line fault model from simple to complex, embedded in the calculation module. When users are operating the simulation module, select the fault model according to their own needs. If users simply want to perform a simple fault calculation, the new module's principle is the same as the traditional simulation calculation principle. If users want to perform complex fault calculation, simply select the corresponding line fault model under the Windows operating interface of the simulation system, then enter the component parameters, click OK to start the calculation and the system will automatically run the embedded EMTP calculation module, generate data automatically into COMTRADE standard format file and send it to the down-position tester. The down-position tester will give feedback about whether the protection device has gone into action, and the results will be given back to the host computer, determine the relevant data follow-up processing. The principle of the test process is shown in Figure 1. 


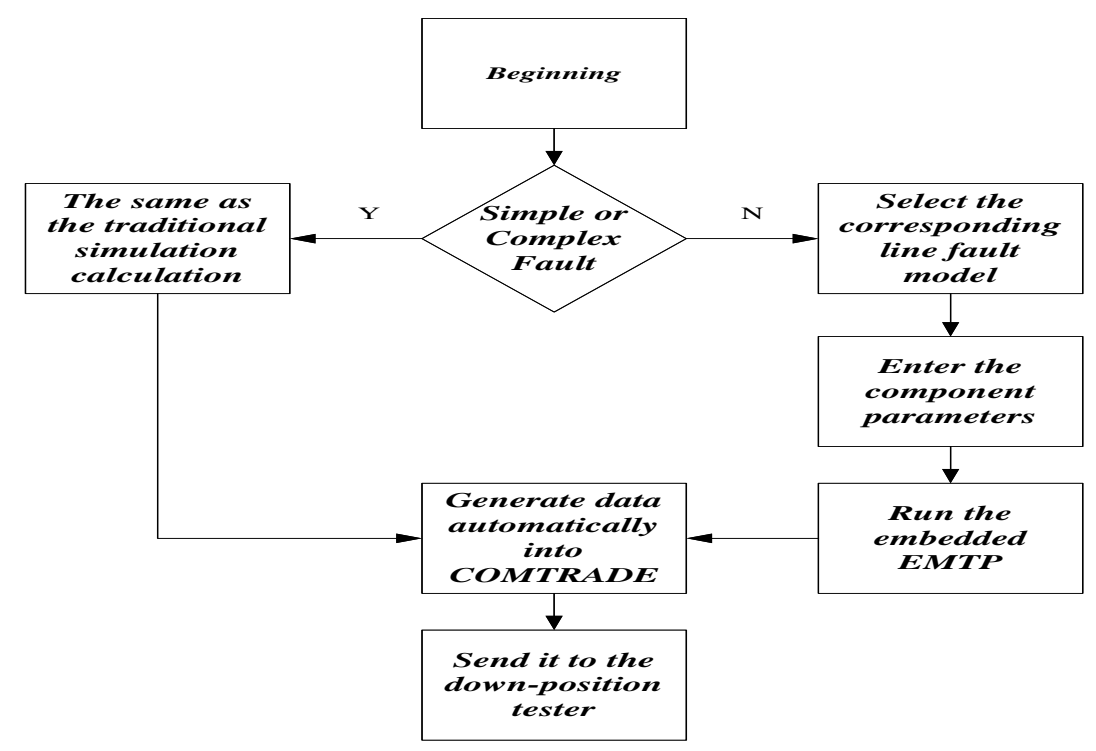

Figure 1 New Simulation Calculation Module Test Flow Chart

The significance of converting data from a file into a COMTRADE standard format is that the down-position tester of digital relay protection testers that are currently common used uses an optical digital interface and the COMTRADE standard format can be easily transferred via the digital interface. It also has the advantages of easy fault playback, easy to read and write the data on other devices and so on. It's an excellent solution to the compatibility of data between different devices. The standard format can also verify the correctness of the protection scheme under system failure or disturbance condition.

\subsection{New calculation module experiment procedure}

Taking the fault of one phase short circuit with one phase break in the power system as an example, the detailed test procedure of the EMTP-based composite fault calculation module is introduced in detail. The fault diagram is shown in Figure 2.

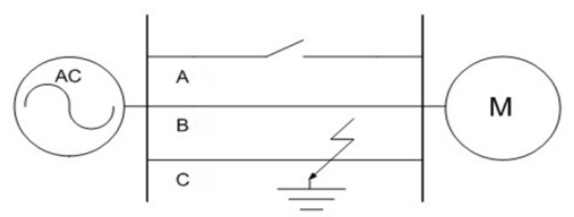

Figure 2 Line Fault Diagram

The new calculation module operation can be divided into the following seven steps:

(1) Select the test type: Open the simulation system software under Windows, select the test type in the selection.

(2) Define protection characteristic parameters.

(3) In the simulation model, select the same line model as the fault line. The model is shown in Figure 3. According to the actual line requirements in the model to fill in the relevant component parameters, and add test items.

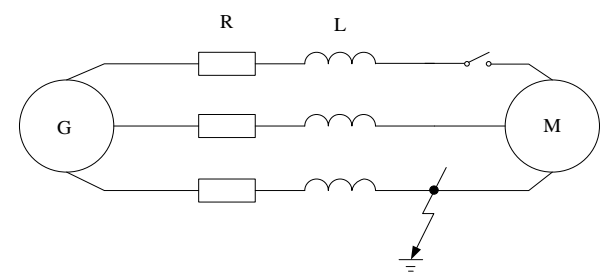

(4) Select the output signal form.

Figure 3 Fault Line Model

(5) Edit the wiring diagram based on hardware configuration.

(6) Select the test report type. 
(7) When the test is started, open the parameter file completed after the above steps. Click OK to start the simulation calculation. The system will automatically run the EMTP-based calculation module embedded in the system.

(8) The simulation results are automatically converted into COMTRADE standard format generated data, sent to the down-position computer, the standard format data conversion results.

The EMTP software is embedded in the new module by rewrite and run under DOS environment. Steps (6), (7) require the testers to finish in the field. The program execution process, through the host computer simulation calculation, will generate information and send it through the interface to the down-position computer of the tester. The down-position tester will give feedback about whether the protection device has gone into action, and the results will be given back to the host computer, determine the relevant data follow-up processing.

\subsection{Comparison between new calculation module and traditional simulation}

The new relay protection tester simulation calculation module is the same with the traditional relay protection tester in hardware, are micro-computers. But the simulation software on the computer is different. The traditional relay protection tester simulation can only perform a simple line fault calculation, the fault simulation principle has been exemplified in previous sections. But the new EMTP-based composite fault real-time simulation system can carry out complex power grid failure calculation that traditional relay protection tester simulation cannot achieve, such as the circuit shown in Figure 3-4, there's one phase grounding short circuit failure along with break failure of another phase.

Fault example shows that for the complex fault of the system, there's one phase grounding short circuit failure along with break failure of another phase, the traditional simulation test cannot calculate the exact formula, therefore it cannot perform accurate simulation calculation. However, in the newly developed calculation module, only to find the corresponding line model in the model library is needed, you can then simulate the failure. The testing process is described in the previous section.

\section{New computing module and RTDS simulation}

\subsection{RTDS performance and testing process}

RTDS means real-time digital simulator, aim for testing the equipment of power system or network response, in order to achieve the study of influence brought by the interference in power system devices and network and to prevent power failure. But RTDS is not dedicated to relay protection test only.

The process of RTDS applied to relay protection simulation test is, first, system modelling by RSCAD and other software, building different test models according to different types of relay protection devices, such as: line relay protection devices, bus relay protection devices, transformer relay protection devices, their simulation test models are different, so the model should be set up separately. The data is compiled by users using the computer after modelling, and the compiled model file is transferred to the down-position computer of the tester to perform high-speed calculation, so as to realize real-time information exchange. The schematic diagram of the test process is shown in Figure 4.

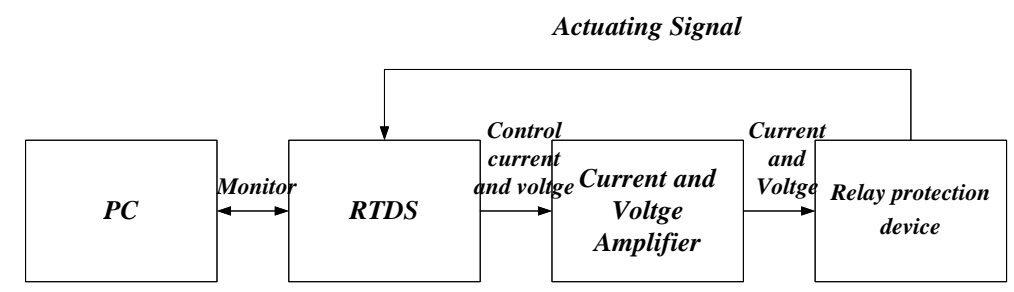

Figure 4 RTDS Test Process Schematic 
RTDS can provide real-time information exchange, real-time means that the simulation output can truly reflect the waveform of real system. The realization principle is that the simulation system can calculate the network at a fast-enough speed so that the simulation data can be continuously output. A well performed real-time exchange can ensure that the simulation test can discover issues of tested devices accurately and in time. RTDS realizes real-time by letting down-position computers to program and calculate its data. But the processing speed of down-position computers' DSP is far slower than host PC. When perform calculation of complex large power grid, RTDS needs to increase the computational nodes to meet the requirements of accuracy. But more nodes mean the increasing of response time, and real-time performance will be more difficult to guarantee. And because of its high cost, the application in industry is limited.

\subsection{Comparison of new calculation module and RTDS simulation}

The new computing module is based on EMTP, so compared with RTDS, both of them have good real-time electromagnetic transient analysis capabilities, rich power system software library and rich simulation test type. However, there're many types of RTDS models, and they are not dedicated to the relay protection test, the operation is relatively time-consuming with high cost. The new calculation module is dedicated to the relay protection test, the model number is relatively small, only the commonly used relay protection model is preserved. The microcomputer is responsible for computing, compared to the RTDS DSP calculation, there is no node problem, so the new computing module in real-time can be guaranteed.

As for the system failures shown in Figure 3-4, RTDS and the new calculation module can both be used to simulate, but the excellent characteristics of RTDS and its broad application decide its expensive price. The new calculation module is based on independent research and development of EMTP, dedicated to electrical protection test simulation calculation, so the price is relatively low compared to RTDS. The new complex fault real-time calculation module of relay protection tester can provide close results to RTDS in relay protection test, but its price is lower, which makes relay protection tester more affordable in industrial practical usage, and enhances product market competitiveness.

\section{Conclusions}

In this paper, we use the EMTP component model library to build a common line fault model. Rewriting in the DOS environment, the EMTP calculation module is embedded in the relay protection tester simulation system. The $\mathrm{C}++$ program is used to realize the automatic conversion of the generated data into COMTRADE format function. In this paper, a new calculation module is proposed for the host computer of the relay protection tester. The new calculation module is based on EMTP, which can calculate the real-time fault of the power system.

The significance of the new computational module is:

Compared with the traditional relay protection tester simulation calculation, it can be used to perform complex multi-point fault calculation. When some simple failures occur, the calculation process is the same as the traditional simulation. When some complex failures occur, the module can run the embedded EMTP software for real-time simulation automatically.

Compared with RTDS, the new simulation module is applied to the relay protection tester, which is dedicated to relay protection only. There is no problem of increasing nodes and ensuring realtime in the calculation of large power grid, which ensures the real-time requirement of the new module.

Compared with RTDS, because the relay protection model is relatively simple, so the gap with RTDS is reduced in the relay protection test, but the price is lower, the reliability of the relay protection tester is improved.

The calculation module is installed in the host computer of the relay protection tester, which has the advantages of realizing the complex fault of the computing system, improving the man-machine interaction process and reducing the requirement of the operator's theoretical knowledge. It can 
improve the market competitiveness of relay protection tester, and achieve the optimal design of relay protection tester.

\section{References}

[1] Wang Z G,Li X J,Wang Y G. (2010) Research on the development of relay protection tester based on unifier building mode[J].Power System Protection and Control.

[2] GurevichV. (2009) Reliability of microprocessor-based relay protection devices:Myths and reality[J]. Serbian Journal of Electrical Engineering, 6(1), 167-186.

[3] ApostolovA. (2006) Object models of protection devices in IEC 61850[C]. Transmission and Distribution Conference and Exhibition, IEEE, 915-920

[4] Li G,Wang C X,Wen B Y. (2005) Research and development of a new experimental device for digital relay protection[J]. Relay, 33(22), 16-20.

[5] Fang S,Huang M,Li X. (2008) Development of fiber digital relay protection tester based on IEC61850[J]. Relay, 36(8), 23-25. 